\title{
Acidic mammalian chitinase and the eye: implications for ocular inflammatory diseases
}

\author{
Claudio Bucolo ${ }^{1 *}$, Maria Musumeci ${ }^{2}$, Salvatore Musumeci ${ }^{3}$ and Filippo Drago ${ }^{1}$ \\ ${ }^{1}$ Department of Clinical and Molecular Biomedicine, Section of Pharmacology and Biochemistry, School of Medicine, University of Catania, Catania, Italy \\ 2 Department of Hematology, Oncology and Molecular Medicine, Istituto Superiore di Sanità, Rome, Italy \\ ${ }^{3}$ Department of Chemical Sciences, University of Catania, Catania, Italy
}

\section{Edited by:}

Stefania Tacconelli, Università degli

Studi G. D'Annunzio, Italy

Reviewed by:

Stefania Tacconelli, Università degli

Studi G. D'Annunzio, Italy

Satish Ramalingam, Kansas

University Medical Center, USA

${ }^{*}$ Correspondence:

Claudio Bucolo, Department of Clinical and Molecular Biomedicine,

Section of Pharmacology and

Biochemistry, School of Medicine,

University of Catania, Viale A. Doria 6,

95125 Catania, Italy.

e-mail: claudio.bucolo@unict.it
Chitinases have an important role in the defense of organisms against chitin-containing parasites. An acidic mammalian chitinase (AMCase) has been detected in epithelial cells in lung tissue samples taken from patients with asthma as well as in conjunctival epithelium of patients with inflammatory ocular diseases. Particularly, elevated AMCase activity has been observed in ocular tissues of patients with vernal keratoconjunctivitis, seasonal allergic conjunctivitis, and in patients affected by dry eye syndrome. This enzyme is induced via a $T_{H}$ 2-specific, IL-13-dependent pathway. AMCase may thus be a key mediator of IL-13-induced responses in $\mathrm{T}_{\mathrm{H}}$ 2-driven inflammatory ocular diseases.

Keywords: acidic mammalian chitinase, inflammation, allergy, dry eye

\section{INTRODUCTION}

Polysaccharides are present in all organisms, mostly for structural purposes. Chitin is plentiful in the structural coatings of fungi, insects, and parasitic nematodes, but it is not produced in mammals. Chitin, the linear polymer of $\beta-1,4$ linked $\beta-N$ Acetyl-glucosamine (GlcNAc), is the most abundant biopolymer in marine ecosystems and, after cellulose, the second most copious polysaccharide in nature. Since chitin seems not to be made in mammals, it was initially assumed that chitinases would also be absent, being restricted to species that do contain the polymer. However, the host defense against chitin-containing pathogens includes the production of chitinases. Chitinases are endo- $\beta$ 1,4 linked $\beta$ - $N$-Acetyl-glucosaminidase, and recently, two distinct chitinases have been identified in humans, chitotriosidase (Chit) expressed in phagocytes, and an acidic mammalian chitinase (AMCase) expressed in gastrointestinal tract, in lung, and conjunctiva. AMCase, $50 \mathrm{kDa}$ protein, is a member of the glycosyl hydrolase 18 family (EC 3.2.1.14) and has structural similarity (Figure 1) with other glycosyl hydrolase 18 family members, including the lectins $\mathrm{Ym} 1$ and $\mathrm{Ym} 2$, which are expressed in macrophages, human cartilage glycoprotein-39 (HC-gp39), and chitotriosidase (Muzzarelli, 2008). Chit and AMCase show chitinase enzymatic activity, whereas other mammalian chitinases do not possess this activity. Characteristically, the resistance to acidic $\mathrm{pH}$ distinguishes AMCase from Chit, which has its optimum at $\mathrm{pH}$ 6. On the contrary, AMCase has its optimum at $\mathrm{pH} 2$ and 4 in rodents and humans, respectively; AMCase is expressed in different tissues such as stomach, lung, and salivary glands, and appears to be associated with inflammatory diseases (Chou et al., 2006). Chit, has been shown to be expressed together with lysozyme in the human lacrimal gland (Hall et al., 2008). This chitinase produced by macrophages and neutrophils also has a role in innate immunity (van Eijk et al., 2005). Since this chitinase was considered more active in the control of chitin-containing pathogens, the presence of measurable Chit activity in tears appears to support the protection of the eye in both humans and mice, where the chitotriosidase (CHIT) gene is evolutionarily conserved (Gianfrancesco and Musumeci, 2004). In this context, it is interesting to note that Chit, unlike bacterial chitinases, does not appear to have any mucolytic activity (Sanders et al., 2007), and therefore the lacrimal film is not modified in its structure, maintaining the integrity of visual function. Moreover, Hall et al. (2008) found that recombinant Chit does not inhibit bacterial growth and does not synergize with lysozyme on the growth of Gram-positive and Gram-negative bacteria, and the antimicrobial activity appears to be limited only to fungi. AMCase appears to play a key role as first responder of the immune system (Ramanathan et al., 2006), both in the epithelial cells lining the nasal mucosa and other contiguous conjunctival cells. When the epithelial cells are stimulated against non-existent parasites through a mechanism that induces an IL-13 response, they can produce chitinases, which represent a marker of both innate immunity and inflammatory response. In addition to the role in innate immune response, the modulation of AMCase expression by specific inhibitors suggests a new pathway for controlling ocular inflammation. This article seeks to provide a succinct review of ocular inflammation, a rapidly changing area of visual science, focusing on the role of chitinase.

\section{AMCase AND BIOLOGICAL ACTIONS}

One of the most important issues in chitinase biology relates to our almost complete lack of understanding of the functions 


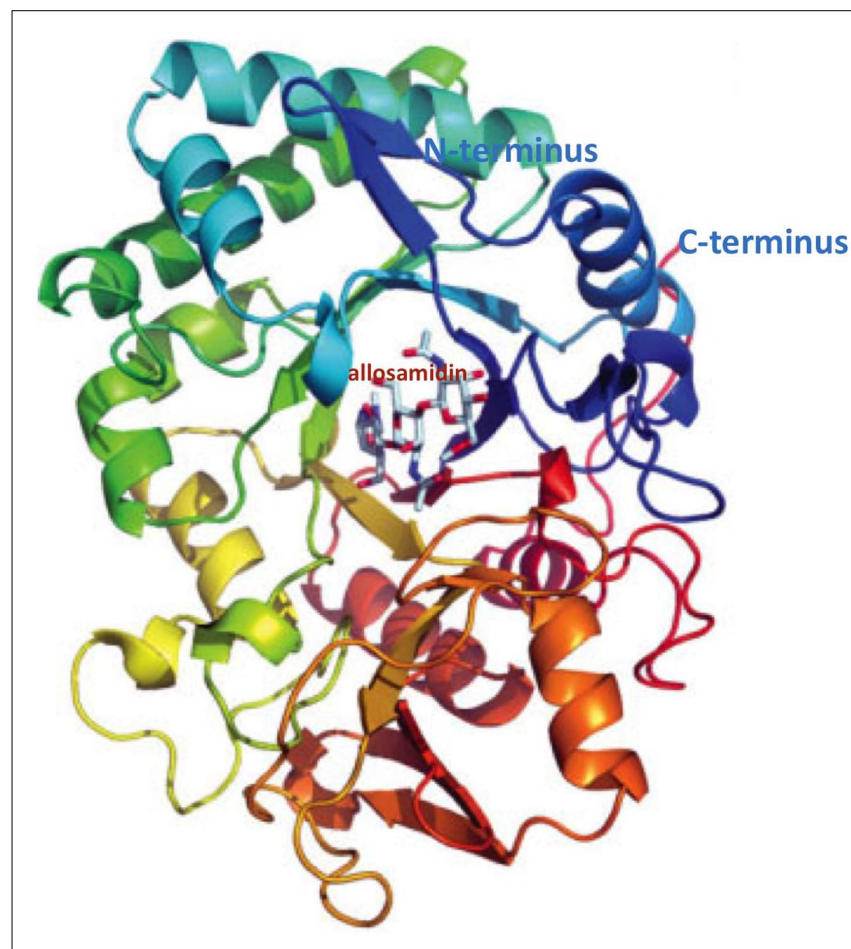

FIGURE 1 | Structure of human AMCase in complex with allosamidin. EC 3.2.1.14; source: EMBL-EBI (European Molecular Biology Laboratory-European Bioinformatics Institute; www.ebi.ac.uk).

of these strongly conserved, and therefore presumably biologically important, enzymes in human subjects. Chitinases have been studied most intensely in lower life forms, where they are produced in significant quantities by hosts defending against infections with chitin-containing organisms. This can be appropriately considered part of the innate immune response against a chitincontaining pathogen. AMCase contains a $30-\mathrm{kDa} \mathrm{N}$-terminal catalytic domain that can hydrolyse chitin, and it has recently been identified both in human gastrointestinal tract and lung. Chitinase production can also play a key role in the life cycle of chitin-containing fungi and parasites, where they control growth and molting, and can be used by pathogens to invade or exploit chitin-containing structures to complete their lifecycle in the host. AMCase represents a product of genes on mouse chromosome 3 and human chromosome 1 at p13.1-p21.3. The enzyme is acid stable, with a $\mathrm{pH}$ optimum of 2.0 , and its appears to be adapted to function in the extreme environment of the stomach where it may play a role in defense and/or digestion of chitin-containing organisms. Recently, Goedken et al. (2011) have demonstrated that AMCase is active with appropriate $\mathrm{pH}$ profile showing that its activity at $\mathrm{pH} 2.0$ was fourfold the activity obtained at higher $\mathrm{pH}$ (5.2 and 7.5). This enzyme might also have non-chitin-related biologic effector properties because an AMCase-like protein has been reported to possess fibroblast growth-promoting activity (Guoping et al., 1997), and AMCase has been implicated in host defenses and food processing (Muzzarelli, 2008). Further, AMCase plays a key role in $\mathrm{T}_{\mathrm{H}} 2$ inflammation (Zhu et al., 2004). AMCase is induced at sites of inflammation (e.g., parasitic infections) and remodeling. This raises the possibility that this enzyme plays active roles in human antiparasite and antiinfective defense and repair responses. Probably AMCase plays a role as sentinel that trigger responses to parasites, infections, and/or antigen challenge, acting directly as chemotactic agents or indirectly by inducing other chemokines that attract eosinophils and $\mathrm{T}$ cells to sites of parasitic infection, in other words AMCase maybe modulate tissue inflammation, immunity, and remodeling (Zhu et al., 2004).

\section{AMCase AS A POTENTIAL THERAPEUTIC MOLECULE}

A role for AMCase in asthma pathophysiology has been suggested following the demonstration that AMCase expression is increased in the lungs of ovalbumin sensitized and challenged mice that develop airway hyperresponsiveness, compared with control animals challenged with phosphate-buffered saline (Zhu et al., 2004). In this animal model of asthma, AMCase is expressed in both airway epithelial cells and alveolar macrophages. Elevated expression of AMCase was also observed in lung tissue from asthmatic patients when compared with normal subjects (Zhu et al., 2004). AMCase is produced in human epithelial cells of lower airways and conjunctiva via a $\mathrm{T}_{\mathrm{H}}$ 2-specific, IL-13-dependent pathway and seems to be associated with asthma and allergic ocular pathologies. AMCase has also been proposed as a potential therapeutic target in $\mathrm{T}_{\mathrm{H}}$ 2-mediated inflammation (Donnelly and Barnes, 2004). In fact, the inhibition of AMCase activity by the chitinase inhibitor allosamidin decreased the number of inflammatory cells in the bronchoalveolar lavage fluid of ovalbumin sensitized and challenged mice, and reduced asthma symptoms (Zhu et al., 2004. Interestingly, the same response was obtained using antisera against AMCase given in the airways by aerosol (Zhu et al., 2004). The understanding of the role of AMCase in allergic disease is only at its beginning and many issues open new possibilities for its control using specific inhibitors of AMCase activity or modulating its expression. In patients with vernal keratoconjunctivitis (VKC) and with seasonal allergic conjunctivitis (SAC) the level of AMCase activity in the tears was found significantly elevated when compare to healthy controls and the highest levels were found in VKC (Musumeci et al., 2008). When RNA was extracted by conjunctival epithelial cells of these patients, quantitative Real Time PCR measurement confirmed that mRNA expression correlates with tear AMCase activity and the expression was significantly higher in VKC and SAC. Also receiver operating characteristic (ROC) analysis demonstrated that the sensitivity and specificity of AMCase measurement were 100\%, addressing the use of AMCase assay in the biochemical diagnosis of VKC and SAC (Musumeci et al., 2008).Recent studies in rabbits, where a uveitis was induced by LPS injection into the eye's anterior chamber, confirmed that increased AMCase activity was measurable in tears and that epithelial cells of conjunctiva express specific mRNA (Bucolo et al., 2008).Further, it was previously demonstrated in experimental model of mouse asthma, the inflammatory reaction induced by LPS was controlled by the chitinase inhibitor and glucocorticoids, instilled at $3 \mathrm{~h}$ interval in conjunctival sac. In dry eye syndrome, another non-allergic 
ocular pathology, an increased AMCase activity was documented and the specific mRNA expressed by epithelial conjunctival cells (Musumeci et al., 2009). In this pathology the eye inflammation can be ascribed to a common mechanism mediated by AMCase, via a $\mathrm{T}_{\mathrm{H}} 2$-specific, IL-13 dependent way. In summary, AMCase may be considered an important mediator in the pathogenesis of $\mathrm{T}_{\mathrm{H}} 2$ inflammation eye's diseases, suggesting its potential diagnostic and therapeutic utility. Inhibition of AMCase, decreasing the inflammation in the mice that overexpressed IL-13, suggests a loop in the modulatory effect of AMCase in allergic asthma as well in allergic conjunctivitis. In fact, AMCase enzyme inhibition with allosamidin does not alter the level of expression of IL-13 receptors, but inhibits IL-13 from stimulating the expression of several chemotactic factors, typical of allergic inflammation and asthma (Romagnani, 2002; Zhu et al., 2002). We showed that, in endotoxin-induced uveitis in rabbit, the production of AMCase in tears was modulated by glucocorticoids treatment and the inhibition of activity by allosamidin, a chitinase inhibitor, caused an effective reduction of the inflammation clinical score (Bucolo et al., 2008). These data are in accordance with the findings generated from other laboratories, Homer et al. (2006) observed that expression of AMCase is upregulated in response to allergen exposure or IL-13-induced inflammation in mouse lung and allosamidin strongly inhibits AMCase. Furthermore, recently Matsumoto et al. (2009) showed that allosamidin and demethyl allosamidin, showed a potent anti-inflammatory activity in allergic mouse models, even though the mechanism of action remains unclear. As mentioned before, we found (Musumeci et al., 2008) an overexpression of AMCase mRNA in epithelial conjunctival cells during VKC and SAC, suggesting that the activity of AMCase could be used as a differentiate parameter for diagnosis (AMCase activity higher in VKC than in SAC). In these studies, it was clear that AMCase did not directly induce a $\mathrm{T}_{\mathrm{H}} 2$ cytokine response, but mediated or modulated the effector response of IL-13 (a cytokine produced by $\mathrm{T}_{\mathrm{H}} 2$ cells).Moreover, it seems that AMCase activity is required for the increased production of the chemoattractants of the inflammatory cells such as monocyte chemoattractant proteins (MCPs) and eotaxins. From these considerations, it seems evident that the expression of AMCase and the $\mathrm{T}_{\mathrm{H}}$ 2-induced inflammation are driven by a sequential mechanism that is attenuated by inhibitors of chitinase (Figure 2).

\section{CONCLUSION}

Increases in acidic mammalian chitinase levels are a potentially important downstream effect of IL-13 stimulation in $\mathrm{T}_{\mathrm{H}} 2$ oriented immune responses to pathogens and parasites AMCase

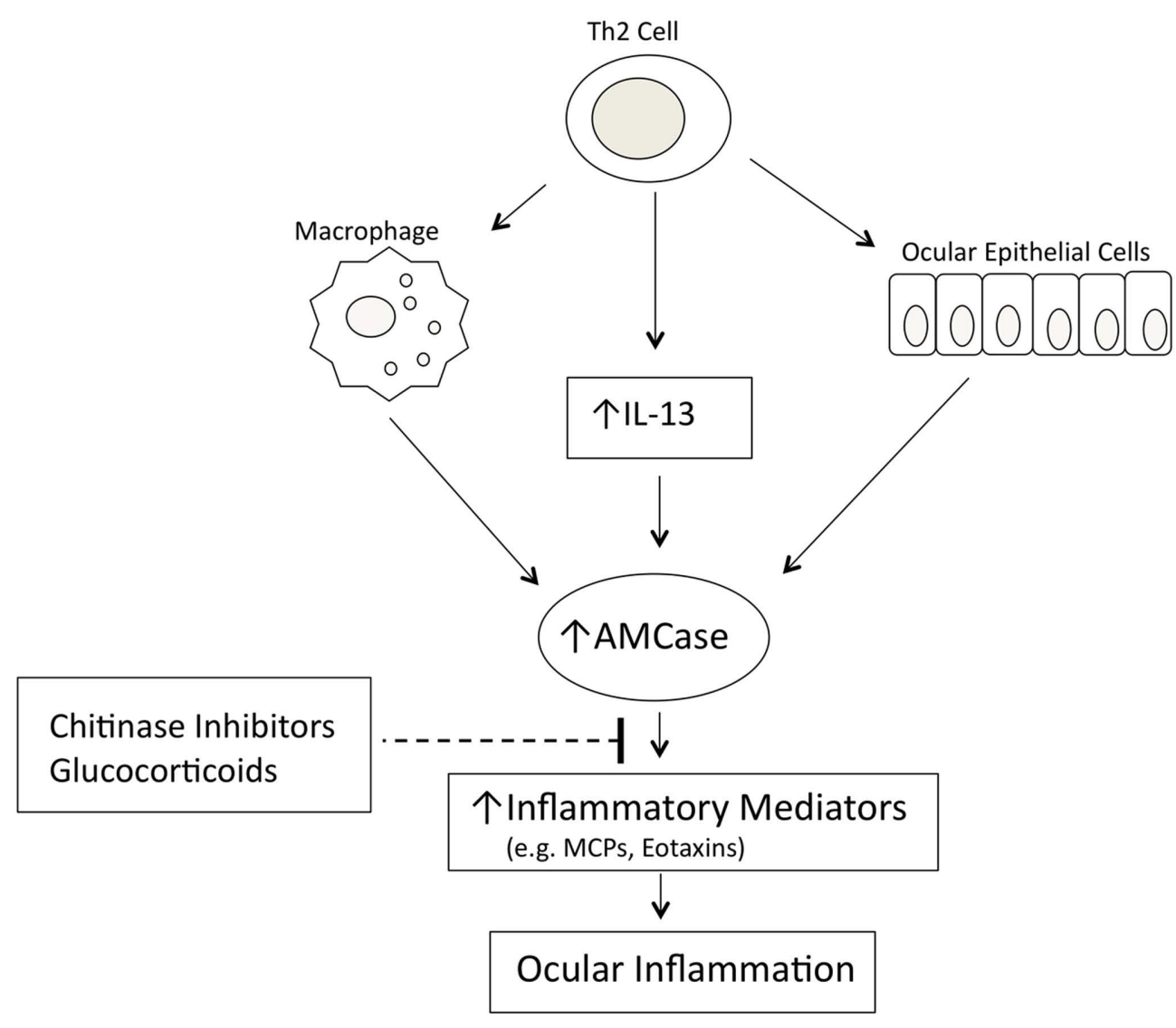

FIGURE 2 | Model of AMCase-mediated ocular inflammation. 
seems to be a key mediator of IL-13-induced responses in $\mathrm{T}_{\mathrm{H}} 2$ driven immune-inflammatory eye diseases with a lofty implication in ocular allergy conditions as well as in dry eye syndrome. However, the immuno-regulatory function of AMCase is not completely clear so far, therefore translational research may be warranted in order to elucidate that.

\section{REFERENCES}

Bucolo, C., Musumeci, M., Maltese, A., Drago, F., and Musumeci, S. (2008). Effect of chitinase inhibitors on endotoxin-induced uveitis in rabbits. Pharmacol. Res. 57, 247-252.

Chou, Y. T., Yao, S., and Czerwinski, R. (2006). Kinetic characterization of recombinant human acidic mammalian chitinase. Biochemistry 45, 4444-4454.

Donnelly, L. E., and Barnes, P. J. (2004). Acidic mammalian chitinase - A potential targetfor asthma therapy. Trends Pharmacol. Sci. 25, 509-511.

Gianfrancesco, F., and Musumeci, S. (2004). The evolutionary conservation of the human chitotriosidase gene in rodents and primates. Cytogenet. Genome Res. 105, 54-56.

Goedken, E. R., O’Brien, R. F., Xiang, T. Banach, D. L., Marchie, S. C., Barlow, E. H., Hubbard, S., Mankovich, J. A., Jiang, J., Richardson, P. L., Cuff, C. A., and Cherniack, A. D. (2011). Functional comparison of recombinant acidic mammalian chitinase with enzyme from murine bronchoalveolar lavage. Protein Expr. Purif. 75, 55-62.

Guoping, C, Fan, P., Jingxi, S, Xiaoping, L., Shiqin, J., and Yuri, L. (1997). Purification and characterization of a silica-induced bronchoalveolar lavage protein with fibroblast growth-promoting activity. J. Cell Biochem. 67, 257-264.

Hall, A. J., Morroll, S., Tighe, P., Götz, F., and Falcone, F. H. (2008). Human chitotriosidase is expressed in the eye and tear gland and has an antimicrobial spectrum different from lysozyme. Microbes Infect. 10, 69-78.

Homer, R. J., Zhu, Z., Cohn, L., Lee, C. G., White, W. I., Chen, S., and Elias, J. A. (2006). Differential expression of chitinases identify subsets of murine airway epithelial cells in allergic inflammation. Am. J. Physiol. Lung Cell. Mol. Physiol. 291, 502-511.

Matsumoto, T., Inoue, H., Sato, Y., Kita, Y., Nakano, T., Noda, N., EguchiTsuda, M., Noriwaki, A., Kan-O, K., Matsumoto, K., Shimizu, T., Nagasawa, H., Sakuda, S., and Nakanishi, Y. (2009). Demethylallosamidin, a chitinase inhibitor, suppresses airway inflammation and hyperresponsiveness. Biochem. Biophys. Res. Commun. 390, 103-108.

Musumeci, M., Aragona, P., Bellin, M., Maugeri, F., Rania, L, Bucolo, C., and Musumeci, S. (2009). Acidicmammalianchitinase (AMCase) in dry eyeconditions. Cornea 28, 667-672.

Musumeci, M., Maltese, A., Bucolo, C., and Musumeci, S. (2008). Chitinase

\section{ACKNOWLEDGMENTS}

The authors gratefully acknowledge support from a grant of the Ministry of Education, University and Research (MIUR), PRIN 2007, and by the International PhD Program in Neuropharmacology, Medical School, University of Catania, Catania, Italy.

levels in the tears of subjects with ocular allergies. Cornea 27, 168-173.

Muzzarelli, R. A. (2008). "New aspects of chitin chemistry and enzymology," in Binomium Chitin-Chitinase Recent Issues, eds S. Musumeci and M. G. Paoletti (New York, NY: Nova Science Publ.), 1-25.

Ramanathan, M. J., Lee, W. K., and Lane, A. P. (2006). Increased expression of acidic mammalian chitinase in chronic rhinosinusitis with nasal polyps. Am. J. Rhinol. 20, 330-335.

Romagnani, S. (2002). Cytokines and chemoattractants in allergic inflammation. Mol. Immunol. 38, 881-885.

Sanders, N. N., Eijsink, V. G., and van den Pangaart, P. S. (2007). Mucolytic activity of bacterial and human chitinases. Biochim. Biophys. Acto 1770, 839-846.

van Eijk, M., van Roomen, C. P., and Renkema, G. H. (2005). Characterization of human phagocyte-derived chitotriosidase, a component of innate immunity. Int. Immunol. 17, 1505-1512.

Zhu, Z., Ma, B., and Zheng, T. (2002). IL-13-induced chemokine responses in the lung: role of CCR2 in the pathogenesis of IL-13-induced inflammation and remodeling. $J$. Immunol. 168, 2953-2962.
Zhu, Z., Zheng, T., Homer, R. J., Kim, Y. K., Chen, N. Y., Cohn, L., Hamid, Q., and Elias, J. A. (2004). Acidic mammalian chitinase in asthmatic $\mathrm{T}_{\mathrm{H}} 2$ inflammation and IL13 pathway activation. Science 304 , 1678-1682.

Conflict of Interest Statement: The authors declare that the research was conducted in the absence of any commercial or financial relationships that could be construed as a potential conflict of interest.

Received: 14 June 2011; accepted: 12 July 2011; published online: 25 July 2011.

Citation: Bucolo C, Musumeci M,

Musumeci $S$ and Drago F (2011) Acidic mammalian chitinase and the eye: implications for ocular inflammatory diseases. Front. Pharmacol. 2:43. doi: 10.3389/fphar.2011.00043

This article was submitted to Frontiers in Inflammation Pharmacology, a specialty of Frontiers in Pharmacology.

Copyright (C) 2011 Bucolo, Musumeci, Musumeci and Drago. This is an openaccess article subject to a non-exclusive license between the authors and Frontiers Media SA, which permits use, distribution and reproduction in other forums, provided the original authors and source are credited and other Frontiers conditions are complied with. 